\title{
recildunds
}

Revista Científica Mundo de la Investigación y el Conocimiento

Jessica Karina Borja Arias a ; Karol Natalie Galarza Ávila ${ }^{\text {b }}$ L Luis Javier Álvarez Moyon ${ }^{\text {c; }}$ Andrea del Consuelo Torres Guzmán ${ }^{\text {d }}$

Tratamiento antiplaquetario prequirúrgico y postquirúrgico

Pre-surgical and post-surgical antiplatelet therapy

Revista Científica Mundo de la Investigación y el Conocimiento. Vol. 3 núm.3. Esp., noviembre, ISSN: 2588-073X, 2019, pp. 906-918

DOI: $10.26820 /$ recimundo/3.(3.Esp).noviembre.2019.906-918

URL: http://recimundo.com/index.php/es/article/view/636

Código UNESCO: 3205 Medicina Interna

Tipo de Investigación: Artículo de Revisión

(C) RECIMUNDO; Editorial Saberes del Conocimiento, 2019

Recibido: 15/09/2019

Aceptado: 23/10/2019

Publicado: 30/11/2019

Correspondencia: karinaborjaa@gmail.com

a. Médico; Investigadora Independiente; Guayaquil, Ecuador; karinaborjaa@ gmail.com

b. Médico; Investigadora Independiente; Guayaquil, Ecuador; karito-naty@ hotmail.com

c. Médico; Investigador Independiente; Guayaquil, Ecuador; lujam898989@ gmail.com

d. Médico; Investigador Independiente; Guayaquil, Ecuador; andytg_17@ hotmail.com 


\section{Tratamiento antiplaquetario prequirúrgico y postquirúrgico}

Vol. 3, núm. 3 Esp., (2019)

Jessica Karina Borja Arias; Karol Natalie Galarza Ávila; Luis Javier Álvarez Moyon; Andrea del

Consuelo Torres Guzmán

\section{RESUMEN}

El tratamiento con medicamentos antiplaquetarios constituye una de las bases fundamentales en los síndromes coronarios agudos. Utilizados tanto para la prevención como para el tratamiento de las enfermedades cardiovasculares (ECV). Por otra parte, la terapia antiplaquetaria también puede ser usada en pacientes durante y después de un procedimiento de angioplastia o colocación de stents, tras un bypass coronario, entre otras. Este tratamiento a pesar de contribuir en la reducción de los eventos isquémicos locales y a distancia, presenta como evento adverso el sangrado, de allí la importancia de la valoración de estos factores ante un procedimiento quirúrgico. El presente estudio permitió revisar y plasmar las consideraciones perioperatorias del uso de antiplaquetarios como parte del tratamiento trombótico en pacientes con ECV. El diseño de investigación que se llevó a cabo es de tipo documental o bibliográfico. Se encontró entre los principales factores a evaluar en la toma de decisión de suspender o mantener el tratamiento antiplaquetario, el tiempo de evolución hasta la intervención, el tipo de presentación, las características clínicas del paciente y el tratamiento a realizarse. Asimismo, en función de la combinación de estos factores, clasifican el riesgo trombótico en alto, moderado o bajo. Las sugerencias y/o procedimientos para el manejo perioperatorio del tratamiento antiplaquetario están basados básicamente en opiniones de expertos, así como en estudios randomizados, razón por la cual pueden encontrarse en la actualidad discrepancias entre algunas guías. No obstante, existen recomendaciones generales y la consideración minuciosa y especial de cada caso, por ser único, con la finalidad de elegir la estrategia que beneficie más al paciente.

Palabras Claves: Tratamiento; Antiplaquetario; Prequirúrgico; Postquirúrgico, Cardiovascular. 


\title{
Tratamiento antiplaquetario prequirúrgico y postquirúrgico
}

Vol. 3, núm. 3 Esp., (2019)

Jessica Karina Borja Arias; Karol Natalie Galarza Ávila; Luis Javier Álvarez Moyon; Andrea del Consuelo Torres Guzmán

\begin{abstract}
Treatment with antiplatelet drugs constitutes one of the fundamental bases in acute coronary syndromes. Used for both prevention and treatment of cardiovascular diseases (CVD). On the other hand, antiplatelet therapy can also be used in patients during and after an angioplasty or stenting procedure, after a coronary bypass, among others. This treatment, despite contributing to the reduction of local and distance ischemic events, presents as an adverse event the bleeding, hence the importance of assessing these factors before a surgical procedure. The present study allowed us to review and capture the perioperative considerations of the use of antiplatelet agents as part of thrombotic treatment in patients with CVD. The research design that was carried out is documentary or bibliographic. It was among the main factors to be evaluated in the decision to suspend or maintain the antiplatelet treatment, the time of evolution until the intervention, the type of presentation, the clinical characteristics of the patient and the treatment to be performed. Also, depending on the combination of these factors, they classify thrombotic risk as high, moderate or low. Suggestions and / or procedures for the perioperative management of antiplatelet therapy are basically based on expert opinions, as well as randomized studies, which is why discrepancies between some guidelines can now be found. However, there are general recommendations and the careful and special consideration of each case, being unique, with the purpose of choosing the strategy that benefits the patient most.
\end{abstract}

Keywords: Treatment; Antiplatelet; Pre-surgical; Post-surgical; Cardiovascular. 


\section{Tratamiento antiplaquetario prequirúrgico y postquirúrgico}

Vol. 3, núm. 3 Esp., (2019)

Jessica Karina Borja Arias; Karol Natalie Galarza Ávila; Luis Javier Álvarez Moyon; Andrea del

Consuelo Torres Guzmán

\section{Introducción.}

La principal causa de muerte a nivel mundial son las enfermedades cardiovasculares (ECV). Se registra en todo el mundo que cada año mueren más personas por ECV que por cualquier otra enfermedad. Se estima que en el año 2015 murieron por esta causa 17,7 millones de personas, esto representa un $31 \%$ del total de las muertes registradas a nivel mundial. De estas cifras, unos 7,4 millones de defunciones fueron por causa de cardiopatía coronaria y unos 6,7 millones de personas murieron como consecuencia de accidentes cerebrovasculares (AVC). Muchas veces el tratamiento requiere de costosas intervenciones quirúrgicas. (Organización Mundial de la Salud, 2017).

En este sentido, se puede aseverar que la prevalencia de enfermedad cardiovascular y la tasa de mortalidad asociada, después de los 45 años, van en ascenso. Asimismo, se ha registrado que la avanzada edad representa un factor que aumenta la incidencia de operaciones no cardíacas. Por otra parte, alrededor de 1/3 de los 25 millones de procedimientos quirúrgicos que se practican cada año, corresponden a pacientes con ECV. "Las muertes cardíacas explican alrededor de $40 \%$ de la mortalidad perioperatoria, la misma proporción que la sepsis, aunque en muchos casos la causa de muerte es insuficiencia de órgano multisistémica”. (Shah \& Crawford, 2015).

Las plaquetas conocidas como los pequeños fragmentos de glóbulos sanguíneos que el cuerpo humano usa para la formación de coágulos y detener hemorragias, juega un papel fundamental en las ECV y los tratamientos asociados. Cuando se encuentran presentes demasiadas plaquetas o en aquellos casos en que se unen demasiado, la persona está propensa a 


\section{Tratamiento antiplaquetario prequirúrgico y postquirúrgico}

Vol. 3, núm. 3 Esp., (2019)

Jessica Karina Borja Arias; Karol Natalie Galarza Ávila; Luis Javier Álvarez Moyon; Andrea del Consuelo Torres Guzmán

formar coágulos, este evento puede ocurrir dentro de las arterias y provocar un ataque cardíaco o un $\mathrm{ACV}$. "La terapia antiplaquetaria hace que sus plaquetas no se unan con tanta frecuencia y ayuda a prevenir la formación de coágulos sanguíneos en sus arterias”. (Enciclopedia Médica ADAM, 2018).

El tratamiento con medicamentos antiplaquetarios constituye una de las bases fundamentales en los síndromes coronarios agudos. Utilizados tanto para la prevención como para el tratamiento de las ECV. Por otra parte, la terapia antiplaquetaria también puede ser usada en pacientes durante y después de un procedimiento de angioplastia o colocación de stents, tras un bypass coronario, entre otras.

Al respecto, la Sociedad Argentina de Cardiología, (2017) refiere que la gran parte de los eventos clínicos agudos y crónicos los cuales influyen en la morbimortalidad son ocasionados por fenómenos trombóticos, los cuales originan embolias que producen isquemia en territorios distantes. En tal sentido, el tratamiento antitrombótico (anticoagulantes y antiplaquetarios) constituye la base sólida para el tratamiento de las enfermedades cardiocirculatorias y la prevención de complicaciones. Sin embargo, el tratamiento antitrombótico a pesar de contribuir en la reducir los eventos isquémicos locales y a distancia, presenta como evento adverso el sangrado. En razón de lo anterior, la efectividad de un tratamiento antitrombótico va a depender, por un lado, del equilibrio entre la reducción de eventos isquémicos y trombosis del stent y, por otro, del sangrado. (p. 5).

El presente estudio permitió revisar y plasmar las consideraciones perioperatorias del uso de antiplaquetarios como parte del tratamiento trombótico en pacientes con ECV. 


\section{Tratamiento antiplaquetario prequirúrgico y postquirúrgico}

Vol. 3, núm. 3 Esp., (2019)

Jessica Karina Borja Arias; Karol Natalie Galarza Ávila; Luis Javier Álvarez Moyon; Andrea del

Consuelo Torres Guzmán

\section{Materiales y Métodos,}

La presente investigación se desarrolló mediante el uso de computadores personales con conexión a internet, a los fines de exponer con relación al manejo perioperatorio de pacientes con tratamiento antiplaquetario. En tal sentido, este trabajo se clasifica como un diseño de investigación documental o bibliográfico. La investigación estuvo limitada a la búsqueda y revisión sistemática de material bibliográfico que, por una parte, es accesible mediante el uso de diversas bases de datos, tales como: PubMed, MedlinePlus, Biblioteca Virtual de la Salud (BVS), SciELO, Sociedad Argentina de Cardiología y Organización Mundial de la Salud (OMS).

Las expresiones o descriptores utilizadas para la búsqueda fueron las siguientes: "antiplaquetarios", "tratamiento antiplaquetario"; "tratamiento trombótico" y "manejo perioperatorio del tratamiento antiplaquetario", el resultado de los registros fue filtrado bajo los criterios de idioma español, correlación temática, relevancia y fecha de publicación en los últimos cinco años.

\section{Resultados.}

\section{Manejo perioperatorio del tratamiento antiplaquetario}

Para Rodríguez et al. (2016) es necesario iniciar con la comprensión de la forma de suspender y reiniciar los antitrombóticos previo a intervenciones quirúrgicas o procedimientos intervencionistas, tomando en cuenta factores tales como las características farmacodinámicas y farmacocinéticas del medicamento usado, además de tener en cuenta las comorbilidades de los 


\section{Tratamiento antiplaquetario prequirúrgico y postquirúrgico}

Vol. 3, núm. 3 Esp., (2019)

Jessica Karina Borja Arias; Karol Natalie Galarza Ávila; Luis Javier Álvarez Moyon; Andrea del Consuelo Torres Guzmán

pacientes y aspectos relativos a la cirugía, como si esta es electiva o de emergencia. El riesgo del sangrado del procedimiento también es un factor importante a considerar. (p. 137).

Vivas et al. (2018) amplían la consideración de estos riesgos, al incorporar en la definición del riesgo trombótico los siguientes aspectos: el tiempo de evolución hasta la intervención, el tipo de presentación, las características clínicas del paciente y el tratamiento realizado. Asimismo, en función de la combinación de estos factores, clasifican el riesgo trombótico en alto, moderado o bajo. "De las variables que evalúa el riesgo trombótico, el tiempo transcurrido entre el episodio isquémico y la intervención es el principal determinante de eventos relacionados con la suspensión de la doble anti agregación plaquetaria (DAP) durante la cirugía o el procedimiento". (p. 555-558).

Al respecto, Nazar, Contreras, Molina \& Fuentes (2018) aportan, específicamente en casos de cirugía electiva no cardiaca de pacientes usuarios de aspirina y clopidogrel, que es importante clasificar al paciente, según el riesgo que presenta ante la cirugía por el uso del antiplaquetario, de la siguiente manera:

1. Pacientes con riesgo trombótico bajo:

- Riesgo hemorrágico bajo: mantener aspirina y suspender clopidogrel 5 días previos a la cirugía.

- Riesgo hemorrágico moderado-alto: suspender aspirina 5-7 días previos al procedimiento quirúrgico y clopidogrel 5 días antes de la cirugía. La suspensión de este fármaco 7 días antes a la cirugía se debe a que diariamente se reponen un $10-12 \%$ de las plaquetas circulantes. 


\section{Tratamiento antiplaquetario prequirúrgico y postquirúrgico}

Vol. 3, núm. 3 Esp., (2019)

Jessica Karina Borja Arias; Karol Natalie Galarza Ávila; Luis Javier Álvarez Moyon; Andrea del

Consuelo Torres Guzmán

2. Pacientes con riesgo trombótico moderado:

- Riesgo hemorrágico bajo-moderado: mantener aspirina y suspender clopidogrel 5 días previos a la intervención quirúrgica.

- Riesgo hemorrágico alto: suspender aspirina 5-7 días previos al procedimiento quirúrgico y clopidogrel 5 días antes de la cirugía.

3. En pacientes con riesgo trombótico alto se debe intentar posponer cirugías electivas hasta una situación de, al menos, riesgo trombótico moderado. Sin embargo, si el procedimiento quirúrgico no es diferible se debe valorar el riesgo de sangrado quirúrgico:

- Riesgo hemorrágico bajo: mantener aspirina y clopidogrel.

- Riesgo hemorrágico moderado: si han transcurrido menos de 6 semanas de un evento trombótico agudo/implante de stent no medicado, o menos de 6 meses de la instalación de un stent medicado, la recomendación es mantener aspirina y clopidogrel. Sin embargo, si han transcurrido más de 6 semanas de un evento trombótico agudo/implante de stent no medicado, o más de 6 meses de la instalación de un stent medicado, la recomendación es mantener aspirina y suspender clopidogrel 3-5 días previos al procedimiento quirúrgico.

- Riesgo hemorrágico alto: mantener aspirina y suspender clopidogrel 5 días previos a la cirugía. En estos pacientes se sugiere iniciar terapia puente con inhibidores de la glicoproteína IIb/IIIa (tirofiban) mientras el paciente no esté recibiendo clopidogrel. El tirofiban se debe iniciar a dosis de $0,1 \mathrm{mcg} / \mathrm{kg} / \mathrm{min}$ y se debe suspender 6 h previo al procedimiento quirúrgico. (p. 294, 295). 


\section{Tratamiento antiplaquetario prequirúrgico y postquirúrgico}

Vol. 3, núm. 3 Esp., (2019)

Jessica Karina Borja Arias; Karol Natalie Galarza Ávila; Luis Javier Álvarez Moyon; Andrea del Consuelo Torres Guzmán

Para (Xavier, 2017) en los casos de pacientes con stent, la discontinuidad de la terapia antiplaquetaria precoz se asocia con un alto riesgo de trombosis del stent y muerte. No obstante, continuar con la medicación también puede significar un alto riesgo de hemorragia en cirugía. En cuyo caso es fundamental evaluar el riesgo trombótico vs el riesgo hemorrágico, de la siguiente manera:

Riesgo trombótico

- Bajo riesgo:> de 6 meses luego de intervencionismo percutáneo-coronario (PCI) con stent metálico (BMS). > de 12 meses luego de PCI con stent con droga (DES).

- Riesgo intermedio: >1 mes < 6 meses luego de PCI con BMS. >6 meses <12 meses luego de PCI con DES.

- Alto riesgo: < de 1 mes luego de PCI con BMS. < 6 meses de PCI con DES. < 12 meses de PCI con DES en casos de stents largos, múltiples, pequeños vasos, bifurcaciones y otras situaciones especiales.

Riesgo Hemorrágico

- En casos de riesgo de hemorragia intermedio, en un riesgo trombótico bajo e intermedio se sugiere continuar con AAS (antiagregante plaquetario que pertenece a la familia de los inhibidores de la ciclo-oxigenasa plaquetaria), discontinuar el inhibidor del receptor P2Y12 5 días antes y reiniciar en 24-72 horas, con una dosis de carga. Si el riesgo trombótico es alto, la conducta es similar, pero podría ser necesaria una terapia puente con inhibidores de Gp IIb/IIIa. 


\section{Tratamiento antiplaquetario prequirúrgico y postquirúrgico}

Vol. 3, núm. 3 Esp., (2019)
Jessila

Consuelo Torres Guzmán
Alvarez Moyon; Andrea del

- En la cirugía de riesgo de sangrado alto, con riesgo trombótico bajo y/o intermedio, la conducta es similar, al igual que el riesgo trombótico alto donde puede ser necesaria la terapia puente con Gp IIb/IIIa.

- En los casos de cirugía general con riesgo de sangrado bajo, con riesgo de trombosis bajo se sugiere continuar con AAS y discontinuar el P2Y12 5 días antes y reiniciar en 24-72 horas con dosis de carga. En los casos de riesgo de trombosis intermedio y alto la sugerencia es continuar con AAS y P2Y12.

- En el riesgo de hemorragia intermedio, con un riesgo de trombosis bajo e intermedio se sugiere continuar con AAS y discontinuar P2Y12 5 días antes y reiniciar en 24-72 horas con dosis de carga. En el riesgo de trombosis alto la conducta es similar, pero se puede plantear la terapia puente con Gp IIb/IIIa.

- En los casos de riesgo de sangrado alto, en el riesgo de trombosis bajo la sugerencia es discontinuar AAS y P2Y12 y reiniciar en 24-72 horas con una dosis de carga. (p. 239, 240)

Zamora, Nazar, \& Lema (2018) indican que la suspensión de estos fármacos va a depender de la indicación que llevó al paciente a utilizarlo, las consecuencias que esta suspensión podría tener, el riesgo trombótico, riesgo de sangrado quirúrgico, además de otras características de cada paciente en particular. Las características particulares de cada paciente son imprescindibles en la toma de decisiones de la suspensión o continuación del tratamiento antiplaquetario y, por ende, el aumento de eventos trombóticos antes y/o después de la intervención quirúrgica o el aumento del riesgo de sangrado. Por otra parte, es importante mencionar que la evidencia disponible al respecto para la elaboración de las guías clínicas es 


\section{Tratamiento antiplaquetario prequirúrgico y postquirúrgico}

Vol. 3, núm. 3 Esp., (2019)

Jessica Karina Borja Arias; Karol Natalie Galarza Ávila; Luis Javier Álvarez Moyon; Andrea del Consuelo Torres Guzmán

bastante regular, por tanto, se elaboran con bases a la opinión de expertos y existe carencia de estudios que abalen dichas prácticas. (p. 236).

Vásquez, Vesga, \& Hernández (2017) respecto a las escalas de medición del riesgo del uso de la terapia antiplaquetaria en pacientes que serán operados, mencionan que entre las más usadas se encuentra la escala DAPT, la cual establece un puntaje entre -2 a +10 . El significado por puntaje corresponde a:

Un puntaje de alto riesgo para eventos isquémicos corresponde a un valor $>2$, en quienes una prolongación de la DAPT por 30 meses ofreció una reducción del desenlace compuesto de infarto de miocardio/trombosis de stent/eventos cardiovasculares con un número necesario para tratar de 34 , con un incremento discreto en el riesgo de sangrado y un número necesario para causar daño de 272. Por otro lado, un puntaje de riesgo bajo corresponde a un valor <2 y en estos casos se demostró que la prolongación de la terapia DAPT no ofreció ningún beneficio en términos de reducción de riesgo para eventos isquémicos, mientras que sí aumentó de forma significativa la presencia de sangrado mayor con un número necesario para causar daño de 64. (p. 50).

\section{Conclusiones.}

La interrupción prematura del tratamiento anti plaquetario se encuentra asociado al aumento de riesgo de complicaciones cardiovasculares, principalmente, se le vincula con la trombosis del stent. En razón de lo cual, es fundamental la decisión de suspender o mantener esta terapia ya que de ella depende, por un lado, el riesgo de trombosis, y por el otro, el riesgo de hemorragias. 


\section{Tratamiento antiplaquetario prequirúrgico y postquirúrgico}

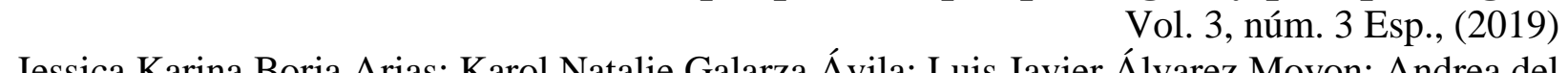

Consuelo Torres Guzmán
Alvarez Moyon; Andrea del

Estas terapias benefician al paciente dada la reducción de episodios trombóticos, sin embargo, también podría perjudicarlo, ya que su evento adverso más negativo es el sangrado. Por tal motivo, es fundamental tomar en cuenta estos dos factores al momento de evaluar su suspensión o continuación ante un procedimiento quirúrgico.

Asimismo, es importante para la toma de decisiones en el manejo de la terapia anti plaquetaria, clasificar el riesgo que presenta el paciente de trombosis y de sangrado, así como del tipo de cirugía, el tiempo de evolución hasta la intervención, el tipo de presentación, las características clínicas del paciente y el tratamiento a realizarse.

Las sugerencias y/o procedimientos para el manejo perioperatorio del tratamiento anti plaquetario están basados básicamente en opiniones de expertos, así como en estudios randomizados, razón por la cual pueden encontrarse en la actualidad discrepancias entre algunas guías. No obstante, existen recomendaciones generales y la consideración minuciosa y especial de cada caso, por ser único, con la finalidad de elegir la estrategia que beneficie más al paciente.

\section{Bibliografía.}

Enciclopedia Médica ADAM. (25 de Agosto de 2018). Medicamentos antiplaquetarios ihibidores P2Y12. Recuperado el 01 de Diciembre de 2019, de MedlinePlus: https://medlineplus.gov/spanish/ency/patientinstructions/000100.htm

Nazar, C., Contreras, J., Molina, I., \& Fuentes, R. (2018). Manejo perioperatorio de pacientes usuarios de antiagregantes plaquetarios. Revista Chilena Cir, 47, 233-239.

Organización Mundial de la Salud. (17 de Mayo de 2017). Enfermedades Cardiovasculares. Obtenido de Organización Mundial de la Salud: https://www.who.int/es/news-room/factsheets/detail/cardiovascular-diseases-(cvds) 


\section{Tratamiento antiplaquetario prequirúrgico y postquirúrgico}

Vol. 3, núm. 3 Esp., (2019)

Jessica Karina Borja Arias; Karol Natalie Galarza Ávila; Luis Javier Álvarez Moyon; Andrea del Consuelo Torres Guzmán

Rodríguez, H., Arauz, A., Asensio, E., Celaya, M., Cordero, A., Guevara, M., . . Rodríguez, G. (2016). Reunión multidisciplinaria sobre el uso de los anticoagulantes orales directos en la fibrilación auricular no valvular. Archivos de Cardiología de México, 87(2), 124-143.

Shah, S., \& Crawford, M. H. (2015). Evaluación y tratamiento del paciente perioperatorio. En M. H. Crawford, Diagnóstico y tratamiento en cirugía cardiaca (Vol. 4, pág. 478). MCGRAW-HILL.

Sociedad Argentina de Cardiología. (Marzo de 2017). Consenso para la Prevención y Manejo del Sangrado en Enfermedades Cardiovasculares. Revista Argentina de Cardiología, 85(1), 57.

Vásquez, S., Vesga, B., \& Hernández, H. (10 de Noviembre de 2017). Antiagregación plaquetaria dual en enfermedad coronaria. Revista Colombiana de Cardiología, 24(S3), 47-55.

Vivas, D., Roldán, I., Ferrandis, R., Marín, F., Roldán, V., Tello, a., . . . Figuero, E. (2018). Manejo perioperatorio y periprocedimiento del tratamiento antitrombotico:documentodeconsensodeSEC,SEDAR,SEACV,SECTCV, AEC,SECPRE,SEPD,SEGO,SEHH,SETH,SEMERGEN,SEMFYC,SEMG, SEMICYUC,SEMI,SEMES,SEPAR,SENEC,SEO,SEPA,SERVEI,SECOT y AEUD. Revista Española de Cardiología, 71(7), 553-564.

Xavier, D. L. (2017). Manejo práctico de agentes antiplaquetarios y terapia puente. Hematología, 21(Extraordinario), 234-243.

Zamora, M., Nazar, C., \& Lema, G. (2018). Nuevos anticoagulantes orales y antiagregantes plaquetarios, en el período perioperatorio de cirugía no cardíaca Parte 2: Nuevos antiagregantes plaquetarios. Revista Chilena de Anestesia, 47, 233-239.

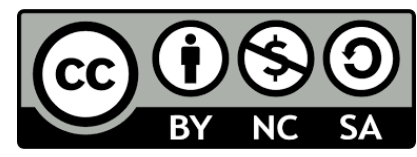

RECONOCIMIENTO-NOCOMERCIAL-COMPARTIRIGUAL

CC BY-NC-SA

ESTA LICENCIA PERMITE A OTROS ENTREMEZCLAR, AJUSTAR Y CONSTRUIR A PARTIR DE SU OBRA CON FINES NO COMERCIALES, SIEMPRE Y CUANDO LE RECONOZCAN LA AUTORÍA Y SUS NUEVAS CREACIONES ESTÉN BAJO UNA LICENCIA CON LOS MISMOS TÉRMINOS. 